\title{
ELECTRIC AND ACOUSTIC ACTIVITY IN NOTCHED FIBER- REINFORCED CONCRETE BEAMS UNDER THREE-POINT BENDING
}

\author{
Dimos TRIANTIS ${ }^{1}$, Dimitri K. TSAOUSI ${ }^{1}$, Ermioni D. PASIOU ${ }^{2}$, Stavros K. KOURKOULIS ${ }^{2}$ \\ 1 Electronic Devices and Materials Laboratory, University of West Attica, Greece \\ E-mail: triantis@uniwa.gr; jimcaushi@gmail.com \\ 2 Laboratory for Testing and Materials, Dept of Mechanics, National Technical University of Athens, Greece \\ E-mail: epasiou@ @eemail.gr; stakkour@central.ntua.gr
}

\section{Introduction}

An experimental study is presented, aiming to comparatively consider the electric and acoustic activity in fiber reinforced concrete beams subjected to three-point bending (3PB). The study is part of an ongoing project attempting to detect phenomena considered as warning signals of upcoming catastrophic fracture, in case brittle materials are loaded mechanically at levels approaching the critical ones.

In this direction, a mature and well established monitoring tool (i.e., Acoustic Emissions - AE) is used, in conjunction with a relatively new sensing technique, based on the detection of very weak electric currents (known as Pressure Stimulated Currents - PSC [1]), produced during the various damage processes preceding and leading up to final macroscopic fracture of brittle materials.

It is concluded that, independently of the nature of the reinforcing fibers, both techniques (i.e., $\mathrm{AE}$ and PSC) follow pretty faithfully the time evolution of the respective mechanical quantities and, what is more important, they provide well distinguishable features considered as pre-failure indicators.

\section{The experimental protocol}

Prismatic concrete beams (length $\mathrm{L}=700 \mathrm{~mm}$ ) of square cross section $\left(150 \times 150 \mathrm{~mm}^{2}\right)$ were subjected to 3 PB with the aid of a stiff servo-hydraulic loading frame. The beams were mechanically notched at their central section. The depth and the width of the notches were equal to $\mathrm{w}=25 \mathrm{~mm}$ and $\mathrm{s}=5 \mathrm{~mm}$, respectively. Three types of specimens were tested: (i) without reinforcement, (ii) with short steel fibers, at a portion of $25 \mathrm{~kg} / \mathrm{m}^{3}$ and (iii) with short plastic fibers, at a portion of $4 \mathrm{~kg} / \mathrm{m}^{3}$ (denoted from here on as "R"-, "M"-- and "P"- type, respectively.

The beams were supported with the aid of two rollers (diameter $\mathrm{d}=30 \mathrm{~mm}$ ) at a distance of $300 \mathrm{~mm}$ from either side of the notch. The load was imposed at the central section with the aid of a third roller of
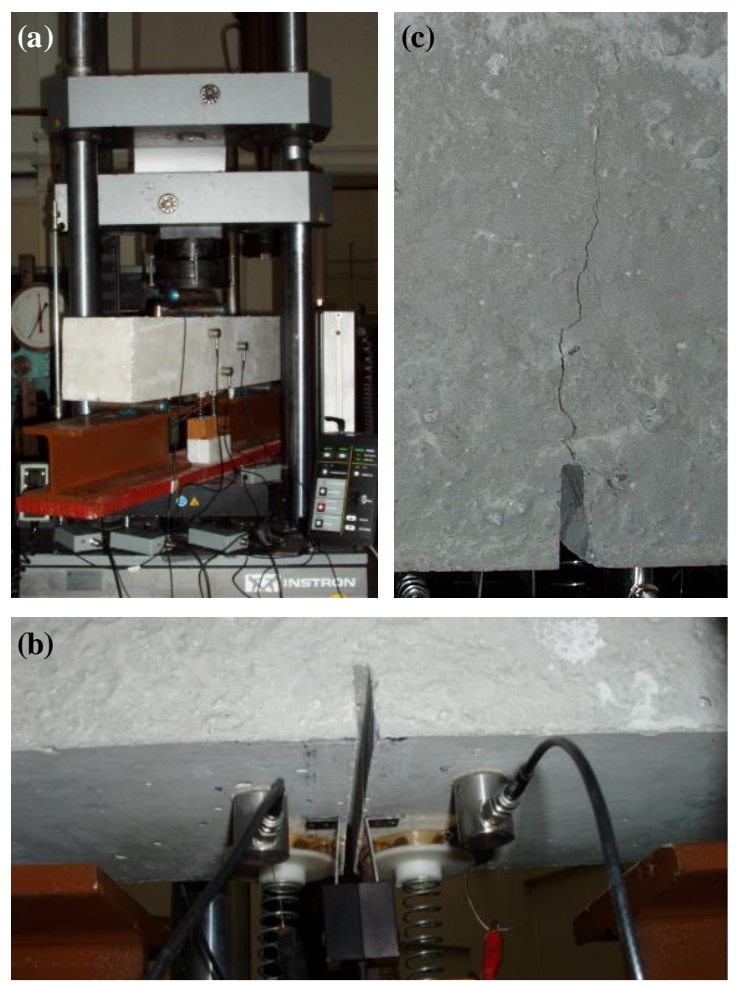

Fig. 1. (a) The experimental set-up; (b) View of the sensors at the lower side of the specimens; (c) Typical slow crack propagation pattern for reinforced beams.

the same diameter. Eight acoustic and two electric sensors, attached at strategic points of the specimens, recorded the acoustic and electric activity. In addition, a clip-gauge was used to record the Notch Mouth Opening Displacement - NMOD.

The load was applied monotonically until fracture, under displacement control mode, at a rate of $0.08 \mathrm{~mm} / \mathrm{min}$ (for NMOD values lower than $1 \mathrm{~mm}$ ) and $0.20 \mathrm{~mm} / \mathrm{min}$ from this NMOD level on.

\section{Experimental results}

The NMOD is plotted as a function of the stress imposed for all three types of specimens in Fig.2. It is seen that while the gain in strength is rather small 

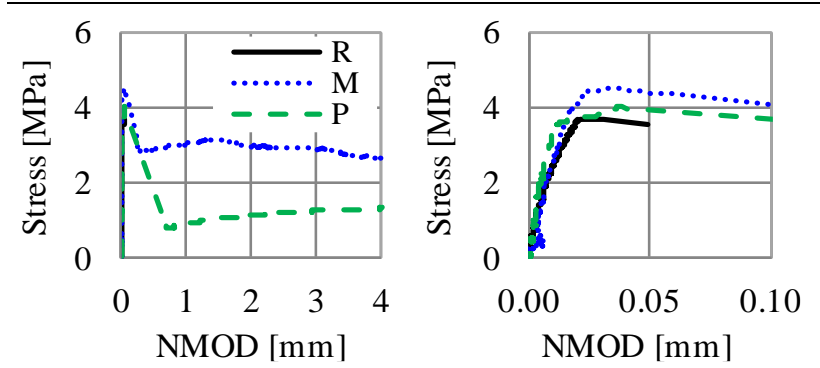

Fig. 2. Effective stress versus NMOD (left plot) and a detailed view for very small NMOD values (right plot).

(especially for the specimens reinforced with plastic fibers) the gain in ductility is huge for both the Mand P-type of specimens.

The PSC recorded is plotted as a function of the normalized (over the duration of the tests) time in Fig.3a. The electric activity for the P-specimens is almost identical to that of the unreinforced ones. On the contrary, the electric activity for the M-type is quite different: the PSC is of much higher intensity and the plot is characterized by a dense network of successive peaks. The acoustic activity, represented by the cumulative number of acoustic hits, is plotted in a semi logarithmic scale in Fig.3b. Again the Rand $\mathrm{P}$-specimens exhibit almost identical behaviour while that of the $\mathrm{M}$-specimens is clearly different.

\section{Discussion and conclusions}

According to the familiar approach for classifying cracking modes, based on the relation between
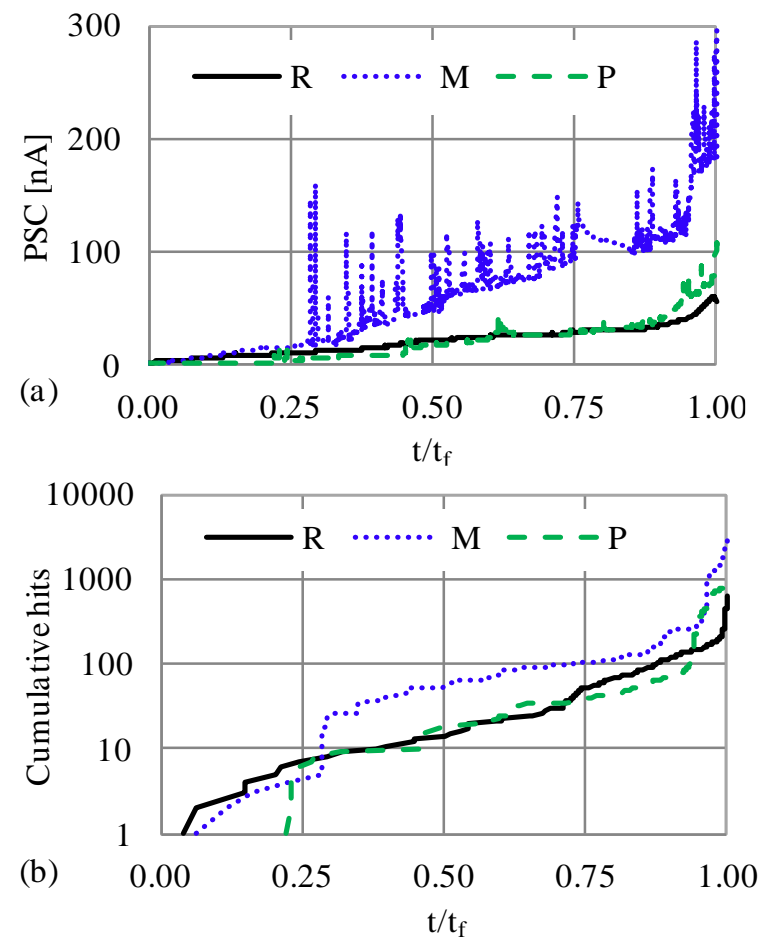

Fig. 3. (a) Electric and (b) acoustic activity vs. time.
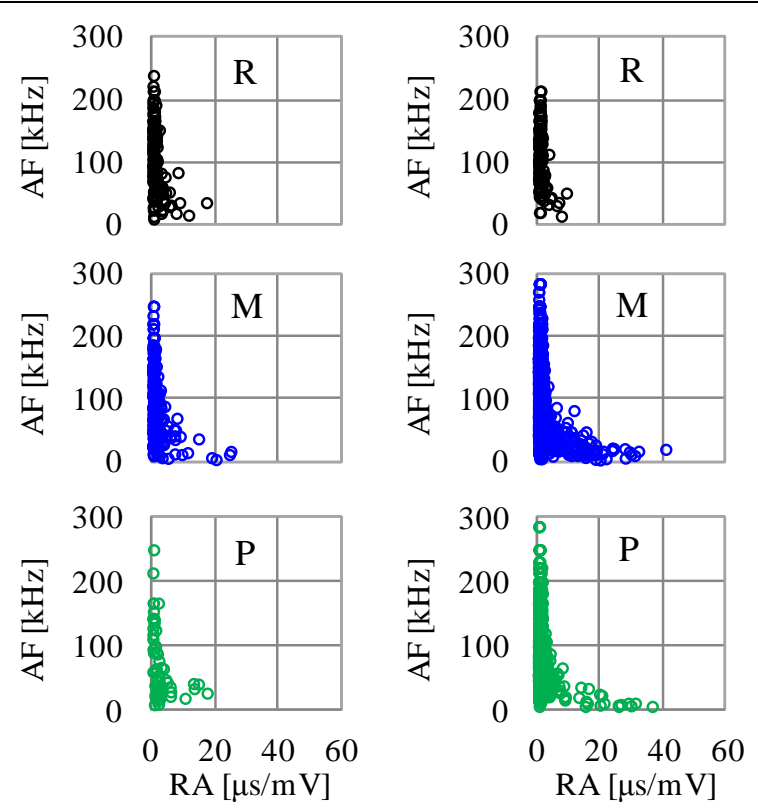

Fig. 4. AF vs. RA (left: early-, right: ultimate-load steps).

the Rise time per Amplitude (RA) and the respective Average Frequency (AF) of the AE signals [2], it can be concluded that: During the first loading steps (up to about $90 \%$ of the fracture load) the cracking mode is almost exclusively tensile (Fig.4, left column). For loads exceeding $90 \%$ of the fracture load events of shear nature (almost $5 \%$ of the total events detected) appear (Fig.4, right column).

The time evolution of the cumulative AE energy and the PSC is shown in Fig.5 for a P-specimen. The similarity of the plots is obvious. Moreover, abrupt increases of the AE energy are accompanied by peaks of the PSC, while both quantities exhibit characteristic changes well in advance of fracture.

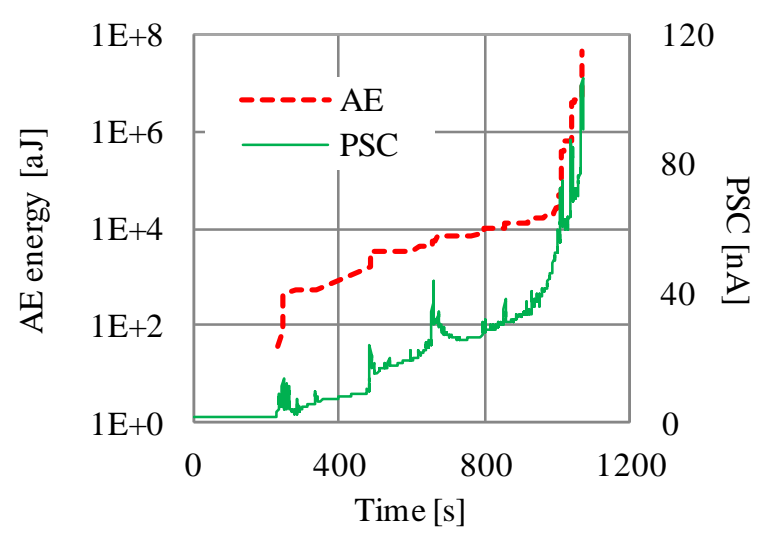

Fig. 5. The cumulative AE energy and the PSC vs. time.

\section{References}

[1] Triantis, D. et al. An analysis of pressure stimulated currents (PSC), in marble samples under mechanical stress. Phys Chem Earth, 2006, 31, 234-239.

[2] Grosse, C.U., Ohtsu, M. Acoustic Emission testing; Springer-Verlag, Heidelberg, Berlin 2008. 